\title{
Wireless Network Code Design and Performance Analysis using
}

\section{Diversity-Multiplexing Tradeoff}

Hakan Topakkaya and Zhengdao Wang, Senior Member, IEEE

\begin{abstract}
Network coding and cooperative communication have received considerable attention from the research community recently in order to mitigate the adverse effects of fading in wireless transmissions and at the same time to achieve high throughput and better spectral efficiency. In this work, we design and analyze deterministic and random network coding schemes for a cooperative communication setup with multiple sources and destinations. We show that our schemes outperform conventional cooperation in terms of the diversity-multiplexing tradeoff (DMT). Specifically, it achieves the full-diversity order at the expense of a slightly reduced multiplexing rate. We establish the link between the parity-check matrix for a $(N+M, M, N+1)$ systematic MDS code and the network coding coefficients in a cooperative communication system of $N$ source-destination pairs and $M$ relays. We present two ways to generate the network coding matrix: using the Cauchy matrices and the Vandermonde matrices, and establish that they both offer the maximum diversity order.
\end{abstract}

\section{Index Terms}

Cooperative communication, network coding, outage probability, diversity-multiplexing tradeoff.

\section{INTRODUCTION}

Channel fading is one significant cause of performance degradation in wireless networks. In order to combat fading, diversity techniques that operate in time, frequency or space are

H. Topakkaya and Z. Wang are with the Department of Electrical and Computer Engineering, Iowa State University, Ames, IA, 50011, USA (e-mail: hakan, zhengdao@iastate.edu). 
commonly employed. The basic idea is to send the signals that carry same information through different paths, allowing the receiver to obtain multiple independently faded replicas of the data symbols. Cooperative diversity tries to exploit spatial diversity using a collection of distributed antennas belonging to different terminals, hence creating a virtual array rather than using physical arrays.

In [1] Ahlswede et al. introduced network coding to achieve the max-flow rate for singlesource multicast that could be impossible to achieve by simply routing the data. Since then, network coding has been recognized as a useful technique in increasing the throughput of a wired/wireless network. The basic idea of network coding is that an intermediate node does not simply route the information but instead combines several input packets from its neighbors with its own packets and then forwards it to the next hop. However, since network coding is devised at the network layer, error-free communication from the physical and medium-access layer is usually assumed, which is a simplifying assumption for wireless communications.

Efforts have also been made to apply network coding to the physical layer, e.g. in [10], [23], [24]. Towards that goal, cooperative schemes have been proposed that make use of network coding in a cooperative communication setup, and studies have been conducted to determine whether network coding provides any advantages over existing cooperative communication techniques [2], [5], [12], [17], [20]-[22].

In [12], a network-coded cooperation (NCC) was proposed and its performance was quantified using the diversity-multiplexing tradeoff analysis which was originally proposed for multiple antenna systems in [25]. NCC was shown to outperform conventional cooperation (CC) schemes which includes space-time coded protocols [8] and selection relaying [3]: It requires less bandwidth, and yield similar or reduced system outage probability while achieving the same diversity order. However, these results are based on an optimistic assumption that any destination node should receive the packets that are not intended for it without any error so that the intended packet can be recovered from the xor'ed packet sent by the relay. When this assumption is removed the scheme can no longer achieve the full diversity order of $M+1$, where $M$ is the number of cooperating relays, but only a reduced diversity order of 2 .

In this paper, we propose a network coded cooperation schemes for $N$ source-destination pairs assisted with $M$ relays. The proposed scheme allows the relays to apply network coding on the data it has received from its neighbors using the coefficients from the parity-check matrix of a 
MDS code. A closed form expression for the outage probability is derived. We also obtain the diversity-multiplexing tradeoff performance of the proposed scheme under two different traffic network models: multicast and unicast. Specifically, it achieves a maximum diversity order $M+1$ at the expense of a slightly reduced multiplexing rate. We also propose two different network coding approaches: deterministic and random. We show that our scheme outperforms NCC and $\mathrm{CC}$ in terms of probability of outage.

The rest of the paper is organized as follows. Section $\llbracket$ discusses the system model, description of the proposed scheme. In Section III-A, performance analysis is established using DMT and the main result is presented. Section [IV discusses the network code design. In Section $\nabla$, we discuss unicast, random network coding and selection relaying. In Section VI the performance of the proposed scheme is compared in terms of DMT and average outage probability with the existing schemes in the literature. Section VII contains the conclusions.

\section{System Model}

\section{A. General System Description}

The network studied in the paper is composed of $N$ source-destination pairs denoted as $\left(s_{1}, d_{1}\right), \ldots,\left(s_{N}, d_{N}\right)$, and $M$ relays denoted as $r_{1}, \ldots, r_{M}$ in a single-cell where all the nodes can hear the transmissions of each other as shown in Fig. 1. We assume that each packet is composed of $L$ bits: $b_{i}=\left[b_{i, 1}, b_{i, 2}, \ldots, b_{i, L}\right]$. We divide $b_{i}$ into smaller blocks of equal length $l$ and represent the $k^{\text {th }}$ block $\left[b_{i, k l+1}, b_{i, k l+2}, \ldots, b_{i,(k+1) l}\right], k \in\{1, \ldots, K\}$ a finite-field element $\theta_{i, k} \in \mathbb{F}_{q}$ where $q=2^{l}$ and $K=L / l$. Therefore, each packet is represented as a $K$-tuple $\Theta_{i}=\left[\theta_{i, 1}, \theta_{i, 2}, \ldots, \theta_{i, K}\right] \in \mathbb{F}_{q}^{1 \times K}$; see e.g., [9], [4]. Dividing each packet into small blocks enables us to work with a smaller field size which in return significantly reduces the complexity of the arithmetic operations. This is to be contrasted to the scheme in [7] where the field size is taken to be $q=2^{L}$. We will give a lower bound on the field size in Sec. IV] We consider two different transmission scenarios. In the first scenario, each source node $s_{i}$ is trying to transmit the data packet $\Theta_{i}$ to all the destinations $d_{i}, i=1, \ldots, N$ which is known as the multicast scenario. In the second scenario, each source node $s_{i}$ is trying to transmit the data packet $\Theta_{i}$ to only destination $d_{i}$ and we will refer to this scenario as the unicast scenario. All the nodes are assumed to be equipped with half-duplex (i.e. cannot transmit and receive at the same time) 
single-antennas. Each data packet $\Theta_{i}$ is error control coded and modulated, and transmitted in $T$ time slots.

The channel between any pair of nodes is assumed to be frequency flat fading with additive white Gaussian noise (AWGN). Let $x_{i} \in \mathbb{C}$ denote the transmitted symbols from node $i$ and $y_{j} \in \mathbb{C}$ the received symbols at node $j$. The additive noise $z_{i} \sim \mathbb{C} \mathbb{N}(0,1)$ has independent and identically distributed (i.i.d.) circularly symmetric entries. Let $h_{i, j} \in \mathbb{C}$ denote the instantaneous channel realization. We assume that the channel coefficient $h_{i, j}$ remains constant during the transmission time of a packet. Then, the channel within one block can be written as

$$
y_{j}(t)=\sqrt{\rho} h_{i, j} x_{i}(t)+z_{i}(t), \quad t=1,2, \ldots, T .
$$

where $\rho$ is the average received SNR at the destination. All the transmissions are made with equal power. In the above equation, the transmitter could be any of the sources or relays, the receiver could be any of the relays or destinations, as long as the transmitter and receiver are different (i.e., not the same relay). The channel coefficient $h_{i, j}$ between any two nodes is modeled as i.i.d. with zero-mean, circularly symmetric complex Gaussian random variables with common variance $1 / \beta$. Therefore, $\left|h_{i, j}\right|^{2}$ is exponentially distributed with parameter $\beta \forall i, j$.

A total of $N L$ bits are transmitted by all sources in $(N+M) T$ channel uses, therefore the system rate is $R=N L /[(N+M) T]$ bits per channel use (BPCU). The transmission rate $R_{0}$ for one source or one relay per one packet is fixed, identical, and equal to $R_{0}=L / T=R(N+M) / N$ BPCU.

The instantaneous mutual information of the channel model in (1) when i.i.d Gaussian input is used is given by:

$$
I\left(X_{i} ; Y_{j}\right)=\log \left(1+\left|h_{i, j}\right|^{2} \rho\right)
$$

where $X_{i}$ and $Y_{j}$ denote the transmitted symbol by node $i$ and received symbol by node $j$. We assume that powerful enough channel codes can be applied within each packet such that if $I\left(X_{i} ; Y_{j}\right)>R_{0}$, the packet can be decoded correctly. In case errors occur, we assume they can be detected. This can be realized through cyclic redundancy check (CRC) code or other parity check codes. When $I\left(X_{i} ; Y_{j}\right) \leq R_{0}$, we say that the channel $h_{i, j}$ is in outage. Otherwise, we say that the channel $h_{i, j}$ is operational. Define $\tau=\left[2^{[R(N+M)] / N}-1\right] / \rho$. Since $\left|h_{i, j}\right|^{2}$ is exponentially 
distributed, the outage probability for the channel in (11) is given by:

$$
P_{0}=\operatorname{Pr}\left(I\left(X_{i} ; Y_{j}\right)<R_{0}\right)=\operatorname{Pr}\left(\left|h_{i, j}\right|^{2}<\tau\right)=1-\exp (-\beta \tau) \cong \beta \tau,
$$

where $a(\tau) \cong b(\tau)$ if $\lim _{\tau \rightarrow 0}[a(\tau) / b(\tau)]=1$.

\section{B. Network Coded Cooperation}

Our transmission scheme consists of two stages; see Fig. 2. In the first stage, direct transmissions from the sources to the destinations take place in $N$ orthogonal time slots. Thanks to the broadcast nature of the wireless medium, all the destinations and the relays overhear the transmissions. At the end of the first stage, each relay tries to decode all $N$ packets. Here one of the two strategies is possible:

1) Strategy $\mathscr{A}$ : If a relay can successfully decode all the packets, then it participates in the second stage. Otherwise, it remains silent. In the second stage, the participating relays perform network coding. Specifically, relay $i$ will transmit the linear combination $\sum_{k=1}^{N} \alpha_{i k} \Theta_{k}$.

2) Strategy $\mathscr{B}$ : If a relay can successfully decode at least one packet, then it participates in the second stage. Specifically, if relay $i$ was able to decode the packets correctly from the sources in the set $S_{i}$ where $S_{i} \subseteq\{1, \ldots, N\}$, then it will transmit the linear combination $\sum_{k \epsilon S_{i}} \alpha_{i, k} \Theta_{k}$.

Unless otherwise specified, we study the first case when the Strategy $\mathscr{A}$ is used until Sec. V Strategy $\mathscr{B}$ will be discussed in Sec. $\mathrm{V}$.

\section{Deterministic and Random Network Coding}

We will consider two network coding schemes for the user cooperation: random coding and deterministic coding. In the random coding approach, which we will refer to as Random Network Coded Cooperation $(\mathrm{RNCC})$, relay $r_{i}$ draws $\alpha_{i j}$ randomly from the finite field $\mathbb{F}_{q}$. After the random coefficients are drawn, a new packet is created by making a linear combination of the source data packets using the $\alpha_{i j}$ 's. In the deterministic approach which will be referred to as Deterministic Network Coded Cooperation (DNCC), the coefficients $\alpha_{i j}$ 's are predetermined and they are designed in a way to maximize the probability that the received linear combinations 
are actually decodable at the destination. We will discuss the problem of how to choose these predetermined coefficients in detail in Sec. IV.

In order to express the overall transmitted signal, we define the following matrix:

$$
A:=\left[\begin{array}{cccccc}
1 & \ldots & 0 & \alpha_{1,1} & \ldots & \alpha_{M, 1} \\
\vdots & \ddots & \vdots & \vdots & . & \vdots \\
0 & \ldots & 1 & \alpha_{1, N} & \ldots & \alpha_{M, N}
\end{array}\right]^{T}
$$

where $(\cdot)^{T}$ denotes transpose. Also define the $N \times K$ finite field vector corresponding to the original source packets as $\Theta=\left[\Theta_{1}^{T}, \Theta_{2}^{T}, \ldots, \Theta_{N}^{T}\right]^{T}$. Using matrices $A$ and $\Theta$, we can express the potential transmitted signals by all the $N$ sources and $M$ relays, in that order, as $\Pi=A \Theta$ where

$\Pi \in \mathbb{F}_{q}^{(N+M) \times K}$. Note that $\Pi$ represents the potential transmitted signals, since due to severe fading some of the channels might be in outage and therefore only a subset of packets can be successfully decoded by some relays. Under Strategy $\mathscr{A}$, such relays will not participate in the second stage and the rows of $A$ corresponding to these relays can be considered to be deleted. Under Strategy $\mathscr{B}$, however, only the coefficients in $A$ that correspond to the unsuccessful packets would be zero, as opposed to a whole row being deleted. Note that, from the destination $d_{i}$ 's perspective, some of the channels might also be in outage. We denote the corresponding submatrix of $A$ for destination $d_{i}$ by $A_{i}$ which satisfies $\Pi_{i}=A_{i} \Theta$ where $\Pi_{i}$ denotes all correctly decoded packets at destination $d_{i}$.

\section{Performance AnAlysis}

\section{A. Diversity-Multiplexing Tradeoff}

As mentioned in the introduction, we will investigate the performance of the proposed scheme via diversity-multiplexing tradeoff (DMT). DMT is accepted as a useful performance analysis tool in cooperative systems [3], [12]. For completeness, we give the formal definitions as in [25]. Let $P_{e}^{i}(\rho)$ denote packet error probability of user $i$ at $\operatorname{SNR} \rho$. Define $P_{e}=\min _{i} P_{e}^{i}$, $i=1, \ldots, N$, then a scheme is said to achieve spatial multiplexing gain $r$ and diversity gain $d$ if the data rate is $\lim _{\rho \rightarrow \infty} R(\rho) / \log (\rho)=r$, and the minimum error probability satisfies $\lim _{\rho \rightarrow \infty} \log \left(P_{e}(\rho)\right) / \log (\rho)=-d$. 


\section{B. Main Result}

Next we define a new parameter which plays a key role in the derivation of the outage probability and hence the achieved diversity order. For any integer $i \in[1, \min (m, n)]$, we define the $\Gamma$-rank, $\Gamma_{i}(C)$, of a $m \times n$ matrix $C$ as an integer $\gamma$ such that 1) any collection of $\gamma$ rows of $C$ is at least rank $i$, and 2) there exists a collection of $\gamma-1$ rows of $C$ that has rank $i-1$. Next, we derive the DMT of the system as a function of $\Gamma_{N}(A)$.

Theorem 1. The diversity-multiplexing tradeoff of DNCC with $N$ source-destination pairs and $M$ intermediate relay nodes which choose their linear combination coefficients from the matrix A for multicast using Strategy $\mathscr{A}$ is given by:

$$
d(r)=\left(N+M-\left(\Gamma_{N}(A)-1\right)\right)\left[1-\frac{N+M}{N} r\right], \quad r \in\left(0, \frac{N}{N+M}\right) .
$$

Proof:

1) Multicast: In the multicast problem, the necessary and sufficient condition for destination $d_{i}$ to recover $\Theta_{i}$ is $\operatorname{rank}\left(A_{i}\right)=N$. To analyze the outage probability, we define the following events: $E_{i} \triangleq\left\{\operatorname{rank}\left(A_{i}\right)<N\right\}$, and $E_{i}^{u p} \triangleq\left\{A_{i}\right.$ has at most $\Gamma_{N}(A)-1$ rows $\}$. Notice that, $E_{i} \subset E_{i}^{u p}$ by the first condition in the definition of $\Gamma$-rank. By the second condition in the definition of $\Gamma$-rank, there exist a collection of rows of $A$ that are rank $N-1$. Let $\tilde{A}_{i}$ denote a $\left(\Gamma_{N}(A)-1\right) \times N$ submatrix of $A$ that consists of such rows. Let $F_{m}$ denote the event that $m$ relays fail to receive all the $\Theta_{i}$ 's correctly. Define $E_{i}^{\text {low }} \triangleq\left\{F_{0} \cap\left\{A_{i}=\tilde{A}_{i}\right\}\right\}$. It follows that $E_{i}^{\text {low }} \subset E_{i}$. Notice that the probability that any relay can successfully decode all $N$ packets in the first stage is $P(S)=\prod_{i=1}^{N} \operatorname{Pr}\left(I_{s_{i} r}(X ; Y)>R_{0}\right)=\prod_{i=1}^{N} \exp (-\beta \tau)=\exp (-N \beta \tau)$. As a result,

$$
P\left(F_{m}\right)=\left(\begin{array}{c}
M \\
m
\end{array}\right) P(S)^{M-m}(1-P(S))^{m} .
$$

Having $N$ direct transmission from the sources and $M-m$ transmissions from the relays, each destination can potentially receive and decode $N+M-m$ packets. Let $E(N+M-m, l)$ denote the event that $l$ out of $N+M-m$ channels were operational:

$$
P(E(N+M-m, l))=\left(\begin{array}{c}
N+M-m \\
l
\end{array}\right) P_{0}^{N+M-m-l}\left(1-P_{0}\right)^{l}
$$


where $P_{0}$ is given by (3). Since $E_{i}^{\text {low }} \subset E_{i} \subset E_{i}^{u p}$, using (6) and (7) we have:

$$
P\left(E_{i}\right) \leq P\left(E_{i}^{u p}\right)=\sum_{m=0}^{M} P\left(F_{m}\right) \cdot \sum_{l=0}^{\Gamma_{N}(A)-1} P(E(N+M-m, l))
$$

and

$$
P\left(E_{i}\right) \geq P\left(E_{i}^{\text {low }}\right)=P\left(F_{0}\right) P_{0}^{N+M-\left(\Gamma_{N}(A)-1\right)}\left(1-P_{0}\right)^{\Gamma_{N}(A)-1}
$$

In (8), the first summation stands for the probability of the event that $m$ of the relays fail to receive all $\Theta_{i}$ 's correctly, leaving us with only $M-m$ relays which will participate in the second stage. In total $N+M-m$ transmissions will be made. The destination $d_{i}$ may not be able to recover all $\Theta_{i}$ 's, if only $\Gamma_{N}(A)-1$ or less number of transmissions are successful.

Notice that, as $\rho \rightarrow \infty, \tau \rightarrow 0$. We need to find the following limit:

$$
\lim _{\tau \rightarrow 0} \frac{P\left(E_{i}\right)}{\tau^{N+M-\left(\Gamma_{N}(A)-1\right)}} .
$$

We consider the individual terms in the summations one-by-one and find the term with the smallest order of $\tau$. Observe that $\lim _{\tau \rightarrow 0}(1-P(S))=N \beta$ and $P\left(F_{m}\right) \cong K_{m} \tau^{m}$ where $K_{m}$ is:

$$
\lim _{\tau \rightarrow 0} \frac{P\left(F_{m}\right)}{\tau^{m}}=\left(\begin{array}{c}
M \\
m
\end{array}\right)(N \beta)^{m} .
$$

Similarly, $P(E(k, l)) \cong K_{k, l} \tau^{k-l}$ where $K_{k, l}=\left(\begin{array}{l}k \\ l\end{array}\right) \beta^{k-l}$. The smallest order $\tau$ term happens when $l$ is equal to $\Gamma_{N}(A)-1$. Hence, we have:

$$
\lim _{\tau \rightarrow 0} \frac{P\left(F_{m}\right)}{\tau^{m}} \cdot \frac{P(E(N+M-m, l))}{\tau^{N+M-m-\left(\Gamma_{N}(A)-1\right)}}=K_{m} K_{N+M-m, \Gamma_{N}(A)-1} \triangleq K_{u p},
$$

and

$$
P\left(E_{i}^{u p}\right) \cong K_{u p} \tau^{N+M-\left(\Gamma_{N}(A)-1\right)}=K_{u p}\left(\frac{2^{\frac{N+M}{N} R}-1}{\rho}\right)^{N+M-\left(\Gamma_{N}(A)-1\right)}
$$

Similarly, we can show that $P\left(E_{i}^{\text {low }}\right) \cong K_{\text {low }} \tau^{N+M-\left(\Gamma_{N}(A)-1\right)}$ where $K_{\text {low }}=\beta^{N+M-\left(\Gamma_{N}(A)-1\right)}$. Now, choosing the fixed rate to be $R=r \log \rho$ and substituting into (12), we obtain:

$$
P\left(E_{i}\right) \cong K \rho^{\left(\frac{N+M}{N} r-1\right)\left(N+M-\left(\Gamma_{N}(A)-1\right)\right)}
$$

where $K_{\text {low }} \leq K \leq K_{\text {up }}$, which is the desired result. 
2) Unicast: In unicast we have a different problem: given the received packets $Y_{i}$ at destination $d_{i}$, we would like to recover only $\Theta_{i}$ from the set of linear equations $Y_{i}=A_{i} \Theta$. The error can only happen when the direct link is in outage. Notice that this implies that $A_{i}$ does not contain $e_{i}$ (the $i$ 'th row of the $N \times N$ identity matrix $I_{N \times N}$ ). In this case, a necessary and sufficient condition for $\Theta_{i}$ to be recoverable is that $e_{i} \in \operatorname{span}\left(A_{i}\right)$, where $\operatorname{span}\left(A_{i}\right)$ is the row-space of $A_{i}$. Here, we make another rank definition that will be useful for the proof of the unicast scenario. We define the $\Lambda_{i}$-rank, $\Lambda_{i}(C)$ of a $m \times n$ matrix $C$ as an integer $\lambda$ such that 1$)$ any collection of $\lambda$ rows of $C$ spans a space that contains $e_{i}$ but 2) there exists a collection of $\lambda-1$ rows of $C$ which does not span a space that contains $e_{i}$. Next, we derive the DMT of the system as a function of $\Lambda_{i}(A)$.

Lemma 1. DMT of DNCC for unicast for the $i^{\text {th }}$ destination is

$$
d_{i}(r)=\left(N+M-\left(\Lambda_{i}(A)-1\right)\right)\left[1-\frac{N+M}{N} r\right], \quad r \in\left(0, \frac{N}{N+M}\right) .
$$

Proof: Here we define the following relevant events. $\bar{E}_{i} \triangleq\left\{e_{i} \notin \operatorname{span}\left(A_{i}\right)\right\}, \bar{E}_{i}^{u p} \triangleq\left\{A_{i}\right.$ has at most $\Lambda_{i}(A)-1$ rows $\}$. Notice that, $\bar{E}_{i} \subset \bar{E}_{i}^{u p}$ by the first condition in the definition of $\Lambda_{i}$-rank. By the second condition in the definition of $\Lambda_{i}$-rank, there exist a collection of $\Lambda_{i}(A)-1$ rows of $A_{i}$ that does not span $e_{i}$. Let $\bar{A}_{i}$ denote a $\left(\Lambda_{i}(A)-1\right) \times N$ submatrix of $A_{i}$ that consists

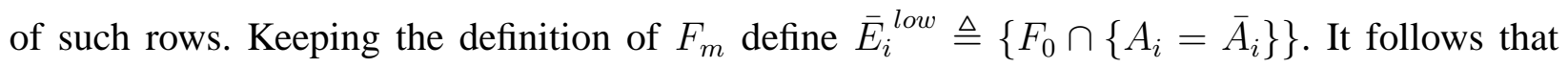
$\bar{E}_{i}^{\text {low }} \subset \bar{E}_{i}$. Since $E_{i}^{\overline{l o w}} \subset \bar{E}_{i} \subset \bar{E}_{i}^{u p}$, using (6) and (7) we have:

$$
P\left(\bar{E}_{i}\right) \leq P\left(\bar{E}_{i}^{u p}\right)=P_{0} \sum_{m=0}^{M} P\left(F_{m}\right) \cdot \sum_{l=0}^{\Lambda_{i}(A)-1} P(E(N-1+M-m, l))
$$

and

$$
P\left(\bar{E}_{i}\right) \geq P\left(\bar{E}_{i}^{\text {low }}\right)=P\left(F_{0}\right) P_{0}^{N+M-\left(\Lambda_{i}(A)-1\right)}\left(1-P_{0}\right)^{\Lambda_{i}(A)-1}
$$

where the first $P_{0}$ in (16) accounts for the outage of the direct link between $s_{i}$ and $d_{i}$. The limits in the second summation in (16) is due to the fact that the destination $d_{i}$ may not be able to recover all $\Theta_{i}$ 's, if only $\Lambda_{i}(A)-1$ or less number of transmissions are successful. The rest of the proof can be completed by showing that the diversity orders of both the upper and the lower bound are equal to (15) as in the proof of the Multicast scenario.

Notice that from the definition of $\Gamma$-rank, we have $\Gamma_{N}(A)=\max _{i} \Lambda_{i}(A)$. Since the error probability $P_{e}$ is defined to be the minimum of individual error probabilities, we have $d(r)=$ 
$\min _{i} d_{i}(r)$. After substituting $d_{i}(r)$ in (15), we obtain the desired result in (5).

Corollary 1. The maximum diversity is achieved if and only if $\Gamma_{N}(A)=N$.

Proof: The result follows immediately from (5).

\section{Design of the Linear Network Coding Matrix}

In this section, we try to design a network coding matrix $A$ that can yield the maximum diversity order. Notice that, by definition we have $\Gamma_{N}(A) \geq N$. Therefore, it is clear from Corollary 1 that we need to pick an $A$ that satisfies $\Gamma_{N}(A)=N$. Before going into the discussion on the design of the matrix $A$, we would like to give another important rank definition that will be used in the design of $A$.

The row Kruskal-rank [15], [18] of $A$, denoted by $\kappa(A)$, is the number $r$ such that every set of $r$ rows of $A$ is linearly independent, but there exist one set of $r+1$ rows that are linearly dependent.

Lemma 2. $\kappa(A)=N \Leftrightarrow \Gamma_{N}(A)=N$.

Proof: We prove $\Gamma_{N}(A)=N \Rightarrow \kappa(A)=N$; the other case is straightforward. When $\Gamma_{N}(A)=N$, from the definition of $\Gamma$-rank any collection of $N$ rows of $A$ is at least rank $N$. But since $\operatorname{rank}(A) \leq N$, we have the first condition for the Kruskal-rank. Also since $\operatorname{rank}(A) \leq N$, any $N+1$ rows will be linearly dependent.

The minimum Hamming distance $d_{\text {min }}$ between any two codewords for a $(n, k)$ error-correcting code is upper bounded by the Singleton bound as $d_{\min } \leq n-k+1$. The codes that achieve this bound are called maximum distance separable (MDS) codes [11]. The following result relates the column Kruskal-rank of the parity-check matrix $H$ of a linear block code to its minimum distance $d_{\min }: d_{\min }=\kappa(H)+1$. This follows from the following theorem in [11] by realizing that $d_{\text {min }}=n-k+1$ for an $[n, k, d] \operatorname{MDS}$ code $\mathscr{C}$.

Theorem 2. ([11] p. 318]) $\mathscr{C}$ is MDS if and only if (iff) every $n-k$ columns of $H$ are linearly independent.

The transpose $H^{T}$ of the parity check matrix of a systematic $(N+M, M, N+1)$ MDS code can be used as an encoding matrix $A$ for our DNCC scheme to minimize the total number of 
packets necessary at the destinations for decoding the source packets. If such an $A$ is used, then each destination needs and only needs $N$ packets (from the sources and relays) for correct decoding. Note that depending on the sizes $N$ and $M$, finding a $(N+M, M, N+1)$ MDS code may or may not be possible in a given finite field $\mathbb{F}_{q}[11]$, [16].

\section{A. Network Code Designs from RS Codes}

Reed-Solomon (RS) Codes are MDS codes. There are two ways of constructing an RS Code: either using the Vandermonde matrices [14] or using the Cauchy matrices [19, Sec. 4.3]. Because of the special structure of $A$ in (4), we will be working on systematic RS codes.

1) Construction based on Cauchy Matrices: The systematic generator matrix for the $\operatorname{RS}(n, k)$ code has the form $G=[I \mid C]$ where $I$ is the identity matrix of order $k$ and $C$ is a $k \times(n-k)$ matrix [19] and $G$ satisfies $\kappa\left(G^{T}\right)=n-k$. $C$ is known as Cauchy matrix and is given by:

$$
C_{i, j}=\frac{u_{i} v_{j}}{x_{i}+y_{j}}, \quad 0 \geq i \geq k-1, \quad 0 \geq j \geq n-k-1 .
$$

where $u_{i}, v_{j}, x_{i}$ and $y_{j}$ are elements of $G F\left(2^{m}\right)$ and are defined as:

$$
\begin{aligned}
& x_{i}=\beta^{n-1-i}, \quad 0 \geq i \geq k-1, \\
& y_{j}=\beta^{n-1-k-j}, \quad 0 \geq j \geq n-k-1, \\
& u_{i}=\frac{1}{\prod_{0 \geq i \geq k-1, l \neq i}\left(\beta^{n-1-i}-\beta^{n-1-l}\right)}, \quad 0 \geq i \geq k-1, \\
& v_{j}=\prod_{0 \geq l \geq k-1}\left(\beta^{n-1-k-j}-\beta^{n-1-l}\right), \quad 0 \geq j \geq n-k-1 .
\end{aligned}
$$

where $\beta$ is the primitive element for $\mathbb{F}_{q}$. Therefore, choosing $n=N+M$ and $k=M$, we construct the network code $A=[I \mid \alpha]$ by choosing $\alpha_{i, j}=C_{i, j}$ which gives $\kappa(A)=N$.

2) Construction based on Vandermonde Matrices: The Vandermonde matrices are defined from a vector of $m$ distinct generating elements $\left\{t_{1}, \ldots, t_{m}\right\}$ of $\mathbb{F}_{q}$ as:

$$
V_{m \times n}:=\left[\begin{array}{ccccc}
1 & t_{1} & t_{1}^{2} & \ldots & t_{1}^{n-1} \\
\cdot & \cdot & \cdot & \ldots & . \\
\cdot & \cdot & . & \ldots & . \\
1 & t_{m} & t_{m}^{2} & \ldots & t_{m}^{n-1}
\end{array}\right] .
$$


The determinant for the square Vandermonde matrix is given by $\left.\operatorname{det}\left(V_{n \times n}\right)=\prod_{1 \leq i<j \leq n}\left(t_{j}-t_{i}\right)\right)$ and $V_{n}$ is nonsingular iff all the $t_{i}$ 's are distinct. To construct $A$ for given $N$ and $M$, we do the following:

1) Choose a suitable $\mathbb{F}_{q}$ with $q=2^{l} \geq N+M$.

2) Choose $N+M$ distinct elements $t_{1}, t_{2}, \ldots, t_{N+M}$ of $\mathbb{F}_{q}$.

3) Construct the Vandermonde matrix $V_{N \times N}$ from $t_{1}, \ldots, t_{N}$ and $V_{M \times N}$ from $t_{N+1}, \ldots, t_{N+M}$.

4) Then $\alpha_{i, j}=\left(V_{M \times N} V_{N \times N}^{-1}\right)_{i, j}$ and $A=\left[I \mid \alpha^{T}\right]^{T}$.

Note that the generating elements that are needed in the construction of RS codes from Vandermonde matrices requires an extra property that they should be the consecutive powers of the primitive element $\beta \in \mathbb{F}_{q}$, i.e. $\beta, \beta^{2}, \ldots, \beta^{2 t}$ for a $t$-error correcting $\mathrm{RS}$ code. We do not need or impose this requirement.

Lemma 3. Let $T \in \mathbb{F}_{q}^{n \times n}$ be an invertible matrix. Then, for any $H \in \mathbb{F}_{q}^{m \times n}$ is the same as that of $H T: \Gamma_{i}(H)=\Gamma_{i}(H T)$ and $\kappa(H)=\kappa(H T), \forall 1 \leq i \leq \min (m, n)$.

Proof: Pick $i \in(1, \min (m, n))$ arbitrary rows from $H$ and denote the resulting $n \times n$ matrix by $H^{\prime}$. We need to show that if the rows of $H^{\prime}$ are linearly dependent or linearly independent then so are the rows of $H^{\prime} T$. But notice that, since $T$ is full-rank we have $x H^{\prime}=0 \Leftrightarrow x H^{\prime} T=0$ where $x \in \mathbb{F}_{q}^{N}$ and $\mathbb{F}_{q}^{N}$ is the $N$-tuples in $\mathbb{F}_{q}$.

Picking $H=\left[V_{N \times N} \mid V_{M \times N}\right]$ and $T=V_{N \times N}^{-1}$ and using Lemma 3$]$ we have $\Gamma_{N}(G)=\kappa(G)=N$. Note that since we need $N+M$ distinct elements of the finite field $\mathbb{F}_{q}$, it is enough to have $q \geq N+M$. Next, we give an example for the case when $N=2$ and $M=2$.

Example: Consider a $[n, k, d]=[4,2,3]$ MDS code $\mathscr{C}$ over $\mathbb{F}_{4}=\left\{0,1, \alpha, \beta=\alpha^{2}=\alpha+1\right\}$ with symbol representations as $\{0=(0,0), 1=(0,1), \alpha=(1,0), \beta=(1,1)\}$. Constructing the Vandermonde matrices from the set $\{0,1, \alpha, \beta\}$ and multiplying with the inverse, we have

$$
V=\left(\begin{array}{cccc}
1 & 1 & 1 & 1 \\
0 & 1 & \alpha & \beta
\end{array}\right)^{T} \quad A=\left(\begin{array}{cccc}
1 & 0 & \beta & \alpha \\
0 & 1 & \alpha & \beta
\end{array}\right)^{T} .
$$

Let $K=1$ and $b_{i}=\left[b_{i, 1}, b_{i, 2}\right], \Theta_{i}=\left[\theta_{i, 1}\right], i=\{1,2\}$. If the above encoder matrix is used, relays will transmit the linear combinations $\beta \theta_{1,1}+\alpha \theta_{2,1}, \alpha \theta_{1,1}+\beta \theta_{2,1}$ respectively. For example for the first relay, this operation will be performed using regular addition in $\mathbb{F}_{2}$ as $\left(b_{1,2}+b_{2,1}+\right.$ $\left.b_{2,2}, b_{1,1}+b_{1,2}+b_{2,1}\right)$ and for the second relay $\left(b_{1,1}+b_{1,2}+b_{2,2}, b_{1,1}+b_{2,1}+b_{2,2}\right)$ which can be 
put in the matrix form as:

$$
\left(\begin{array}{llll}
0 & 1 & 1 & 1 \\
1 & 1 & 1 & 0 \\
1 & 1 & 0 & 1 \\
1 & 0 & 1 & 1
\end{array}\right)\left(\begin{array}{l}
b_{1,1} \\
b_{1,2} \\
b_{2,1} \\
b_{2,2}
\end{array}\right) .
$$

Notice that since $\kappa(A)=2$ and the destination will be able to recover $\Theta_{1}, \Theta_{2}$ when at least two of the transmissions are successful. It is important to emphasize that unlike MDS code construction that we gave earlier, here we do not have any restrictions on the code size for any given $N$ and $M$, we can find a large enough finite field $\mathbb{F}_{q}$ satisfying $q=2^{l}, l \geq N+M$.

\section{Discussions AND FurThER IMPROVEMENTS}

In the previous sections, we have established the DMT of DNCC for a given network coding matrix $A$. Later, we designed the network coding matrix $A$ to have the property that $\Gamma_{N}(A)=$ $\kappa(A)=N$. Here in this section, we look at the performance of DNCC under the Strategy $\mathscr{B}$ and the case when only some of the relays (which are selected according to their channel qualities) are allowed to transmit. We also investigate the performance of the case when the linear combination coefficients are chosen randomly.

\section{A. Strategy $\mathscr{B}$}

Decode-and-forward schemes suffer from performance loss when the source-relay channel is in outage. If a multi-source scenario is considered the performance loss becomes even more severe. Therefore, the assumption that the relay has to decode all the packets in order to be able to cooperate may be too restrictive for such schemes. We could relax this assumption and assume that the relays will participate cooperation even though they have not been able to decode all the packets.

Denote the outage event under Strategy $\mathscr{B}$ by $E_{i}^{\mathscr{B}}$. Notice that under Strategy $\mathscr{B}$, not only the $M-m$ relays as in (6) but also the rest of the $m$ relays contributes to the decoding at the destinations. Clearly the probability of not being able to solve the linear system of equations will decrease and hence the performance will get better, i.e. $P\left(E_{i}^{\mathscr{B}}\right) \leq P\left(E_{i}\right)$. Taking $\Gamma_{N}(A)=N$, the probability of $E_{i}^{\text {low }}$ becomes $P\left(E_{i}^{\text {low }}\right)=P\left(F_{0}\right) P_{0}^{M+1}\left(1-P_{0}\right)^{N-1}$. We have $P\left(E_{i}^{\text {low }}\right) \leq$ 
$P\left(E_{i}^{\mathscr{B}}\right) \leq P\left(E_{i}\right)$. But using a similar analysis as in the proof of Theorem 1, it can be shown that even though Strategy $\mathscr{B}$ offers lower packet error rate, the DMT is the same as that of Strategy $\mathscr{A}$. That is, even though Strategy $\mathscr{B}$ improves the packet error rate performance, the DMT remains unchanged.

\section{B. RNCC:}

In RNCC the linear combination coefficients $\alpha_{i, j}$ 's are chosen randomly from a finite field $\mathbb{F}_{q}$. Similar to the deterministic case, destination $d_{i}$ cannot recover $\Theta_{i}$ when the submatrix $A_{i}$ is rank deficient, i.e. $E_{i}=\operatorname{rank}\left(A_{i}\right)<N$. However, unlike the deterministic case there are two possible reasons to have a rank deficient $A_{i}$ in the random case: one is due to fading and the other is due to the choice of the random coefficients $\alpha_{i j}$ 's. The former happens when at most $N-1$ channels are operational resulting in an $A_{i}$ matrix that has at most $N-1$ rows. Notice that no matter what $\alpha_{i, j}$ 's are chosen $A_{i}$ will be rank deficient and hence the linear system of equations cannot be solved. Therefore, we define this event to be deterministic error event: $E_{i}^{\text {det }}=\left\{A_{i}\right.$ has at most $N-1$ rows $\}$ in the proof of Theorem 11 and probability of this event is

given by $P\left(E_{i}^{\text {det }}\right)=\sum_{m=0}^{M} P\left(F_{m}\right) \cdot \sum_{l=0}^{N-1} P(E(N+M-m, l))$. Notice that $E_{i}^{\text {det }} \subset E_{i}$. On the other hand, due to the random choice, relays may choose linearly dependent coefficients which will result in an $A_{i}$ matrix such that $\operatorname{rank}\left(A_{i}\right)<N$. Denote this event by $E_{i}^{\text {ran }}$. But by the Corollary 1, this event will result in the outage events that have diversity order less than $M+1$. Therefore, the key idea of this proof is to isolate such events, and show that the probability of such events can be bounded by the field size.

$$
\begin{aligned}
P\left(E_{i}\right) & =P\left(E_{i} \mid E_{i}^{\text {det }}\right) P\left(E_{i}^{\text {det }}\right)+P\left(E_{i} \mid E_{i}^{\text {ran }}\right) P\left(E_{i}^{\text {ran }}\right) \\
& =P\left(E_{i}^{\text {det }}\right)+P\left(E_{i} \mid E_{i}^{\text {ran }}\right) P\left(E_{i}^{\text {ran }}\right) \leq P\left(E_{i}^{\text {det }}\right)+P\left(E_{i} \mid E_{i}^{\text {ran }}\right)
\end{aligned}
$$

Next, we present the lemma that upper bounds the term $P\left(E_{i} \mid E_{i}^{r a n}\right)$.

Lemma 4. The probability that any $N \times N$ square submatrix $A_{i}^{\prime}$ of $A_{i}$ is rank deficient is upper bounded by,

$$
P\left(E_{i} \mid E_{i}^{r a n}\right) \leq \frac{N}{q}
$$


Proof: The proof consists of an application of the Schwartz-Zippel Lemma of a carefully chosen error event as in the proof of Theorem 2 in [6]. We skip the proof due to space limitations.

Now, using Lemma 4 we see that if the field size is large enough, the error event will be dominated by the event $E_{i}$ with high probability:

$$
\lim _{q \rightarrow \infty} P\left(E_{i}\right) \leq P\left(E_{i}^{\text {det }}\right)+\lim _{q \rightarrow \infty} \frac{N}{q}=P\left(E_{i}^{\text {det }}\right) .
$$

Note that, although the limit is taken asymptotically with $q \rightarrow \infty$, it is enough to have $q \cong \rho^{M+1}$. The rest of the proof is the same with the above proof for DNCC. Below we summarize all the above proved results with the following theorem:

Theorem 3. DNCC with $M$ intermediate relay nodes which choose their linear combination coefficients from the rows of $A$ that satisfies $\Gamma_{N}(A)=N$ and $N$ source nodes achieves the DMT in both the multicast and unicast scenario and under both strategies $\mathscr{A}$ and $\mathscr{B}$ :

$$
d(r)=(M+1)\left[1-\frac{(N+M)}{N} r\right], r \in\left(0, \frac{N}{N+M}\right) .
$$

RNCC achieves the same DMT as in (29) with probability at least $1-\frac{N}{q}$, where $q$ is the field size.

\section{Selection Relaying:}

We can also consider the case where not all of the $M$ relays transmit, but only $K$ selected relays transmit. The same selection rule based on the instantaneous wireless channel conditions can be adapted as in [12]. Define

$$
h_{i} \triangleq \min \left\{\left|h_{s_{1 i} r_{i}}\right|^{2},\left|h_{r_{i} d_{1 i}}\right|^{2}, \ldots,\left|h_{s_{N} r_{i}}\right|^{2},\left|h_{r_{i} d_{N}}\right|^{2}\right\}
$$

where $h_{i, j}$ is the channel coefficient between node $i$ and node $j$. Then, select the $K$ relays that maximizes $h_{i}$, namely first choose $r$ with the rule: $r=\arg \max _{r_{i}} h_{i}$ and continue the same process of choosing the maximum in the beginning of each relay transmission. Note that this selection mechanism can be implemented using a distributed protocol at the network layer as in [3]: relays choose a timer that is inversely proportional to the quality of their channels. Relays assess the quality of their channels from the RTS-CTS packets that were transmitted by the 
source and destination nodes respectively 1 . No CSI is required at the physical layer. Next, we give the diversity-multiplexing performance of this scheme.

Theorem 4. DNCC scheme with the selection of the best $K$ relay nodes out of $M$ and $N$ source nodes, $\Gamma_{N}(A)=N$ in the multicast scenario achieves the DMT:

$$
d(r)=(K+1)\left(1-\frac{(N+K)}{N} r\right), \quad r \in\left(0, \frac{N}{N+K}\right)
$$

if $K<N-1$, and otherwise achieves the DMT:

$$
d(r)=(N+M(K-(N-1)))\left(1-\frac{N+K}{N} r\right), \quad r \in\left(0, \frac{N}{N+K}\right)
$$

Proof: Let $r=\arg \max h_{i}$ where $h_{i}$ is as in (30). The cdf for $\left|h_{j r}\right|^{2}$ (or $\left|h_{r j}\right|^{2}$ ) where $j$ can be a source (or a destination) node was derived in [12] as:

$$
\begin{aligned}
& F(\tau)=\int_{0}^{\tau} \sum_{m=1}^{M}\left\{\beta_{k r_{m}} e^{-\beta_{m} \phi} \prod_{j \neq m}^{M}\left(1-e^{-\beta_{j}}\right)\right\} d \phi+ \\
& \int_{0}^{\tau} \sum_{m=1}^{M} \int_{o}^{\phi}\left(\beta_{m}-\beta_{m k}\right) e^{-\left(\beta_{m}-\beta_{m k}\right) \theta} \prod_{j \neq m}^{M}\left(1-e^{-\beta_{j} \theta}\right) d \theta \beta_{m k} e^{-\beta_{m k} \phi} d \phi
\end{aligned}
$$

where $\beta_{m, k}$ 's are the parameters of the exponential random variables associated with the corresponding channels between node $m$ and node $k$, and $\beta_{m}=\sum_{k=1}^{N}\left[\beta_{m, k}+\beta_{k, m}\right], m=$ $\{1, \ldots, M\}$. Taking $\beta_{m, k}=\beta$ and using exponential expansion a high-SNR approximation for (33) can be shown to be equal to: $F(\tau) \cong(2 N \beta)^{M-1} \beta \tau^{M}$. Now, the probability that any relay can successfully decode all $N$ packets in the first stage is $P(S)=\prod_{k=1}^{N} P\left(\left|h_{k r}\right|^{2}>\tau\right)=$ $\prod_{k=1}^{N}(1-F(\tau))=(1-F(\tau))^{N}$. Similar to the definition of $F_{m}$, let $F_{k}$ denote the event that $k$ out of $K$ relays fail to receive all the packets: $P\left(F_{k}\right)=\left(\begin{array}{c}K \\ k\end{array}\right) P(S)^{K-k}(1-P(S))^{k}$. Using similar techniques as in the proof of Theorem 1, it can be shown that $\operatorname{Pr}\left(E_{k}\right) \cong K_{1} \tau^{M k}$ where $K_{1}=\left(\begin{array}{l}K \\ k\end{array}\right)(2 N)^{(M-1) k} \beta^{M k} N^{k}$.

Also let $E_{s, t}(k)$ denote the event that $s$ channels out of $N$ source channels and $t$ channels out of $K-k$ relay channels were operational. Then we have $P\left(E_{s, t}(k)\right)=$ $\left(\begin{array}{c}N \\ s\end{array}\right) P_{0}^{N-s}\left(1-P_{0}\right)^{s}\left(\begin{array}{c}K-k \\ t\end{array}\right) F(\tau)^{K-k-t}(1-F(\tau))^{t}$. Notice that, since $\Gamma_{N}(A)=N$ we have $E_{i}=E_{i}^{u p}$. Therefore, we have $P\left(E_{i}\right)=\sum_{k=0}^{K} P\left(F_{k}\right) \cdot \sum_{\{s, t \mid s+t=0\}}^{N-1} P\left(E_{s, t}(k)\right)$. It can be

\footnotetext{
${ }^{1}$ Forward and backward channels between the relays and the destinations are assumed to be the same due to reciprocity theorem [13].
} 
shown that $P\left(E_{s, t}(k)\right) \cong K_{2} \tau^{(N-s)^{++}(K-k-t)^{+} M}$, where $(x)^{+}=\max (x, 0)$ and $K_{2}=$ $\left(\begin{array}{c}N \\ s\end{array}\right)\left(\begin{array}{c}K-k \\ t\end{array}\right) \beta^{N-s+(K-k-t) M}(2 N)^{(M-1)(K-k-t)}$ and hence $P\left(E_{i}\right) \cong K_{1} K_{2} \tau^{M k+(N-s)^{+}+(K-k-t)^{+} M}$. We need to find out $\min _{\{s, t \mid s+t=\{0, \ldots, N-1\}\}}\left((N-s)^{+}+(K-t)^{+} M\right)$. We need to consider two different cases: $K<N-1$ and $K \geq N-1$. For the first case, choosing $t=K$ and $s=N-1-t=N-1-K$ achieves the minimum: $(N-s)^{+}+(K-t)^{+} M=$ $(N-(N-1-K)+(K-K) M=K+1$. And for the second case choosing $t=N-1$ and $s=0$ achieves the minimum: $(N-s)^{+}+(K-t)^{+} M=N+(K-(N-1)) M$. Now, rest of the proof can be completed using similar techniques as in the proof of Theorem 1 .

In [3], for a single source single destination setup it was proved that instead of transmitting from all the $M$ relays, if a selection is performed and only the relay with the best channel coefficient transmits then the BER at the destination enjoys a $M$-fold diversity gain. Inspired by this idea, the authors in [12] proposed a Network-coded cooperation (NCC) scheme for $N$ s-d pairs and $M$ relays where only the "best" relay selected according to (30), XOR'es all the source packets and transmits to the destination (Fig. 2 (b)). However, the $M$-fold diversity order can only be achieved when an unrealistic assumption is made. The assumption is that the destination has to be able to decode all the other source packets successfully. When no such assumption is made, no gain from the selection process is obtained and only a diversity order of one is achieved. The significance of our result in Theorem 4 is that if enough number of relays could be used $(K \geq N-1)$, we can achieve $N+M(K-(N-1))$ diversity order.

\section{COMPARISON WITH OTHER SCHEMES}

\section{A. DMT Comparison}

In this section, we would like to compare diversity-multiplexing tradeoff of the previously proposed schemes in the literature. The closest scheme in the literature is the NCC scheme considered in [12]. In NCC instead of all the relays, only one relay transmits which results in total of $N+1$ time slots. Using fewer time-slots NCC achieves a better spectral efficiency than the proposed scheme here. However, NCC can only provide a fixed diversity order of two, while the the proposed scheme achieves the full-diversity order of $M+1$.

In the following, for comparison we include the DMT performance of NCC and that of conventional cooperation (CC) which includes space-time coded protocols [8] and selection relaying [3]. 
The diversity-multiplexing tradeoff of NCC is given by [12]: $d(r)=2(1-r(N+1) / N), r \in$ $\left(0, \frac{N}{N+1}\right)$. The DMT of the decode and forward strategy with $M$ intermediate relay nodes is given by [8]: $d(r)=(M+1)(1-2 r), r \in(0,0.5)$.

To show the advantage of the proposed schemes, we present diversity-multiplexing tradeoff of the existing schemes and the proposed schemes in Fig. 3. As can be seen from the figure, both of the proposed schemes and CC provide a full diversity order of $M+1$ when $r \rightarrow 0$. However, the proposed schemes can provide a higher diversity gain than $\mathrm{CC}$ when the spectral efficiency increases.

\section{B. System Outage Probability}

Here, we compare the system outage probability of the proposed schemes with the other schemes. The system outage occurs when any $d_{i}$ is unable to decode $\Theta_{i}$ reliably: $P_{s}=1-$ $\prod_{i=1}^{N}\left(1-P\left(E_{i}\right)\right)$. We compare $P_{s}$ with the system outage probabilities (30), (43) derived in [12]. In Fig. 4 for a network consisting of two source-destination pairs with various number of relays is compared. As can be seen from the figure, the proposed method clearly outperforms NCC by achieving the full diversity of $M+1$ as compared to NCC's fixed diversity order of 2. The same performance is observed in Fig. 5 where the number of source-destination pairs is increased to three.

\section{Monte-Carlo Simulation}

Here, we compare these schemes with the existing schemes via Monte-Carlo simulations for various number of relays. In the simulations, only channel conditions are considered to isolate the diversity benefits of the scheme. We generate an $(N+M) \times N$ and an $N \times N$ matrix that contains the channel coefficients for each destination and each relay, respectively. Then, we decide that the transmission is successful for any link if the instantaneous channel condition is large enough to be able to support the given data rate and we update the same size linear coefficient matrices accordingly. After all the transmissions take place, we perform Gaussian elimination on the updated linear coefficient matrices to conclude whether each destination $d_{i}$ was able to recover the source packet $\Theta_{i}$ or not. The channel coefficient variances are chosen to be equal to one and $R_{0}=1 \mathrm{BPCU}$. Please note that we considered the average outage error probability which is found by dividing the total number of errors occurred by the number of 
source nodes instead of the system error probability. Please note that, in all the figures only the unicast scenario is adapted since CC cannot be implemented in a multicast scenario. We compare the proposed schemes in Fig. 6 and Fig. 7 with NCC and CC. As can be seen from the figures, the proposed schemes are able to provide the $M+1$ diversity order and outperform the other schemes.

Theorem 3 claims that the performance loss incurred due to the assumption under Strategy $\mathscr{A}$ is not in terms of diversity gain but it is in terms of coding gain. This is validated through simulations as shown in Fig. 8 and Fig. 9 using Monte-Carlo simulations which are set up in the same way as in Fig. 6 and Fig. 7

\section{CONCLUSIONS}

In this paper, we have proposed two network coded cooperation schemes for $N$ sourcedestination pairs assisted with $M$ relays. We studied two different traffic network models: multicast and unicast. The proposed schemes allow the relays to apply network coding on the data it has received from its neighbors. We allow the relays to linearly combine the packets with coefficients either drawn randomly from a finite field or drawn from a linearly independent set of coefficients. We established the diversity-multiplexing tradeoff performance of the proposed schemes, and showed its advantage over the existing schemes. Specifically, it achieves the fulldiversity order $M+1$ at the expense of a slightly reduced multiplexing rate. The novelty in the proposed scheme is that we establish the link between the parity-check matrix for a $(N+M, M, N+1)$ systematic MDS code and the coefficients to perform network coding in a cooperative communication scenario consisting of $N$ source-destination pairs and $M$ relays. We presented two ways to generate the network coding matrix: using the Cauchy matrices and the Vandermonde matrices.

\section{REFERENCES}

[1] R. Ahlswede, N. Cai, D. N. C. Li, and R. W. Yeung, "Network information flow," IEEE Trans. Inform. Theory, vol. 46, no. 4, pp. 1204-1216, Apr. 2000.

[2] X. Bao and J. Li, "Adaptive network coded cooperation (ancc) for wireless relay networks: matching code-on-graph with network-on-graph,” IEEE Trans. Wireless Communications, vol. 7, no. 2, pp. 574-583, Feb. 2008.

[3] A. Bletsas, A. Khisti, D. P. Reed, and A. Lippman, "A simple cooperative diversity method based on network path selection," IEEE Journal on Selected Areas in Communications, vol. 24, no. 3, pp. 659-672, Mar. 2006. 
[4] G. Drolet, "A new representation of elements of finite fields $\mathrm{GF}\left(2^{m}\right)$ yielding small comlexity arithmetic circuits," IEEE Transactions on Computers, vol. 47, no. 9, pp. 938-946, Sept. 1998.

[5] Z. Han, X. Zhang, and H. Poor, "Cooperative transmission protocols with high spectral efficiency and high diversity order using multiuser detection and network coding," Proc. of IEEE Intl. Conf. on Com., pp. 4232-4236, June 2007.

[6] T. Ho, M. Medard, R. Koetter, D. R. Karger, M. Effros, S. Jun, and B. Leong, "A random linear network coding approach to multicast," IEEE Trans. Inform. Theory, vol. 52, no. 10, pp. 4413-4430, Oct. 2006.

[7] R. Koetter and M. Medard, "An algebraic approach to network coding," IEEE/ACM Transactions on Networking, vol. 11, no. 5, pp. 782-795, Oct. 2003.

[8] J. N. Laneman and G. W. Wornell, "Distributed space-time-coded protocols for exploiting cooperative diversity in wireless networks," IEEE Trans. Inform. Theory, vol. 49, no. 10, pp. 2415-2425, Oct. 2003.

[9] R. Lidl and H. Niederreiter, Introduction to finite fields and their applications, Cambridge University Press, 1986.

[10] R. Louie, Y. Li, and B. Vucetic, "Practical physical layer network coding for two-way relay channels: performance analysis and comparison,” IEEE Trans. Wireless Communications, pp. 764-777, Feb. 2010.

[11] F. J. MacWilliams and N. J. A. Sloane, The Theory of Error-Correcting Codes, Amsterdam: North-Holland, 1977.

[12] C. Peng, Q. Zhang, M. Zhao, Y. Yao, and W. Jia, "On the performance analysis of network-coded cooperation in wireless networks," IEEE Transactions on Wireless Communications, vol. 7, no. 8, pp. 3090-3097, Aug 2008.

[13] T. S. Rappaport, Wireless Communications: Principles and Practice, NJ: Prentice Hall, 1996.

[14] I. S. Reed and G. Solomon, "Polynomial codes over certain finite fields," SIAM Journal of Applied Math., vol. 8, pp. 300-304, 1960.

[15] N. Sidiropoulos, G. Giannakis, and R. Bro, "Blind PARAFAC receivers for DS-CDMA systems," IEEE Trans. Signal Processing, vol. 48, no. 3, pp. 810-823, Mar. 2000.

[16] R. C. Singleton, "Maximum distance Q-Nary codes,” IEEE Trans. Inform. Theory, vol. 10, pp. 116-118, 1964.

[17] T. Wang and G. Giannakis, "Complex field network coding for multiuser cooperative communications," IEEE Journal on Selected Areas in Communications, pp. 561-571, Apr. 2008.

[18] Z. Wang and G. B. Giannakis, "Complex-field coding for OFDM over fading wireless channels," IEEE Trans. Inform. Theory, vol. 49, no. 3, pp. 707-720, Mar. 2003.

[19] S. Wicker and V. Bhargava, Reed-Solomon Codes and Their Applications, Wiley-IEEE Press, 1999.

[20] D. Woldegebreal and H. Karl, "Multiple-access relay channel with network coding and non-ideal source-relay channels," ISWCS, pp. 732-736, Oct. 2007.

[21] M. Xiao and M. Skoglund, "Design of network codes for multiple-user multiple-relay wireless networks," Proc. IEEE Intl. Symp. on Info. Theory, pp. 2562-2566, July 2009.

[22] M. Yu, J. Li, and R. S. Blum, "User cooperation through network coding," Proc. of IEEE Intl. Conf. on Com., pp. 4064-4069, June 2007.

[23] S. Zhang, S. Liew, and P. Lam, "Phsical layer network coding," Annual International Conference on Mobile Computing and Networking (ACM Mobicom), Sept. 2006.

[24] S. Zhang and S.-C. Liew, "Channel coding and decoding in a relay system operated with physical-layer network coding," IEEE Journal on Selected Areas in Communications, pp. 788-796, June 2009.

[25] L. Zheng and D. N. C. Tse, "Diversity and multiplexing: a fundamental tradeoff in multiple-antenna channels," IEEE Trans. Inform. Theory, vol. 49, no. 5, pp. 1073-1096, May 2003. 


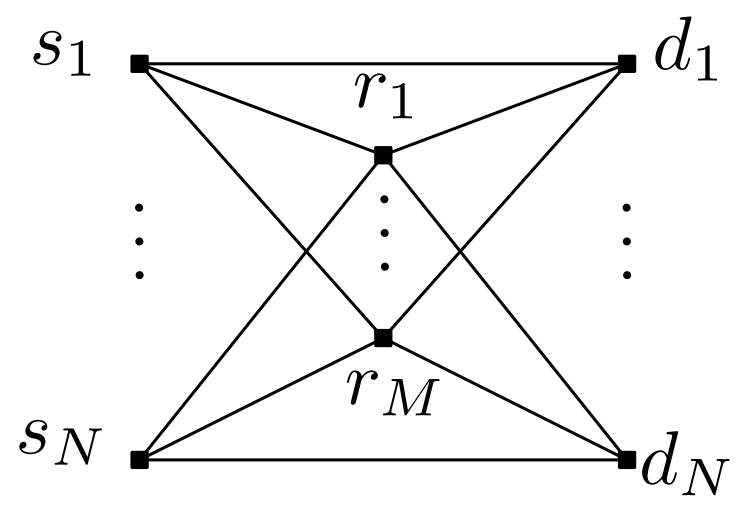

Fig. 1. System model: $N$ source-destination pairs and $M$ relays

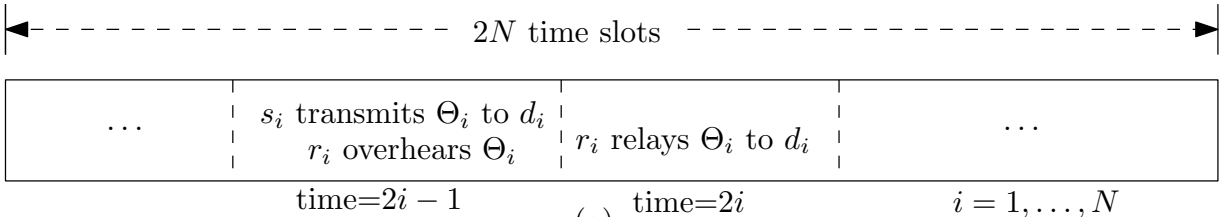

(a)

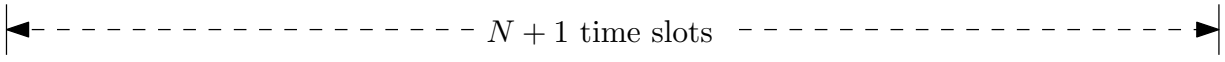

\begin{tabular}{|c|c|c|c|}
\hline$\cdots$ & $s_{i}$ transmits $\Theta_{i}$ to $d_{i}$ & $\cdots$ & $r$ relays $\Theta_{1} \oplus \cdots \oplus \Theta_{N}$ \\
& $r$ and $d$. overhears $\Theta_{i}$ & $\cdots, N$ & time $=N+1$
\end{tabular}

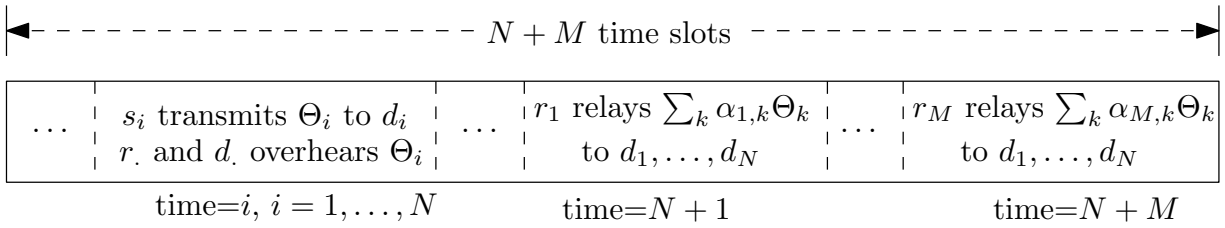

(c)

Fig. 2. time-division allocation for the different schemes compared: (a) CC (b) NCC (c) DNCC, RNCC 


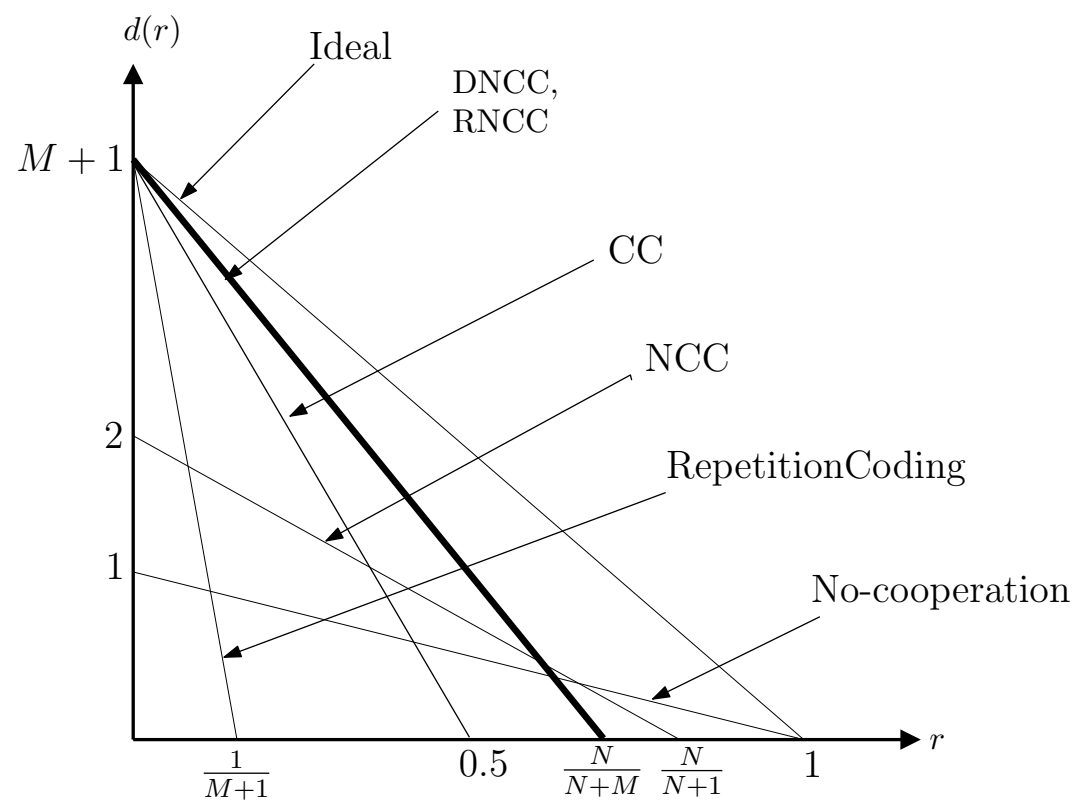

Fig. 3. DMT comparison of various schemes

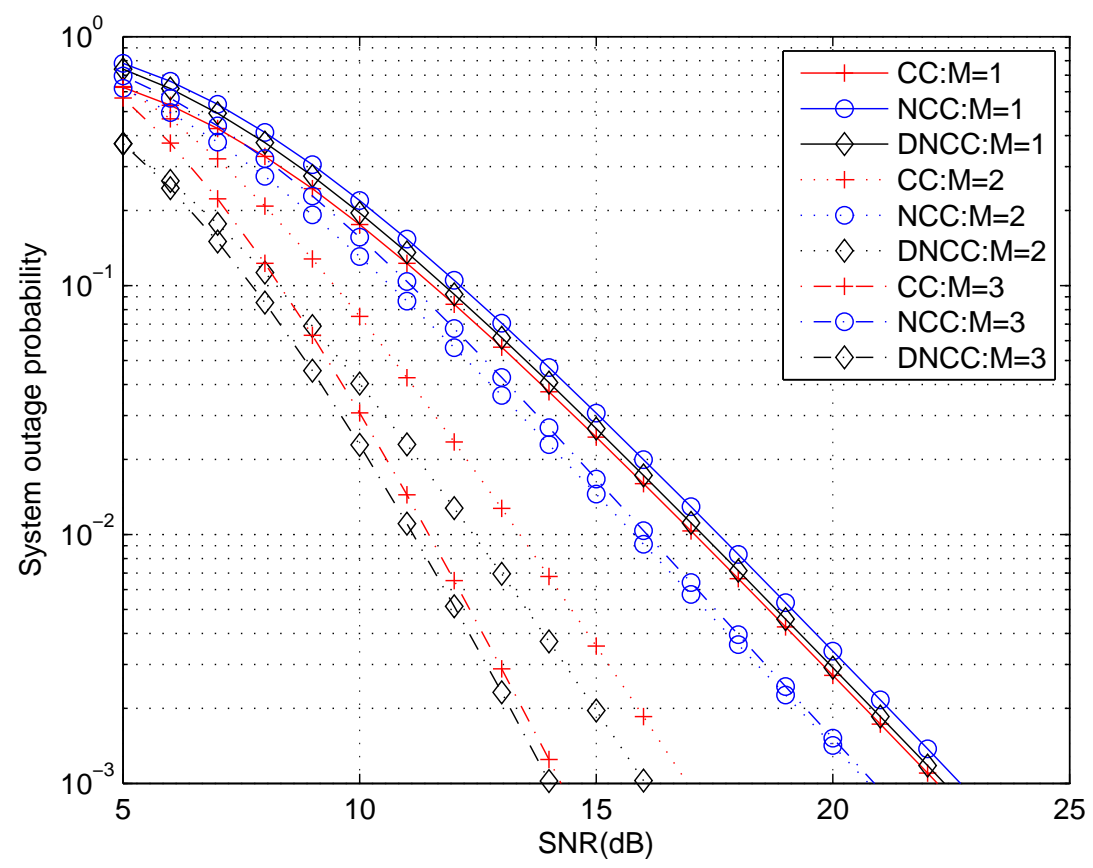

Fig. 4. System outage probability comparison. $(\mathrm{N}=2 \mathrm{M}=1, \ldots 3)$ 


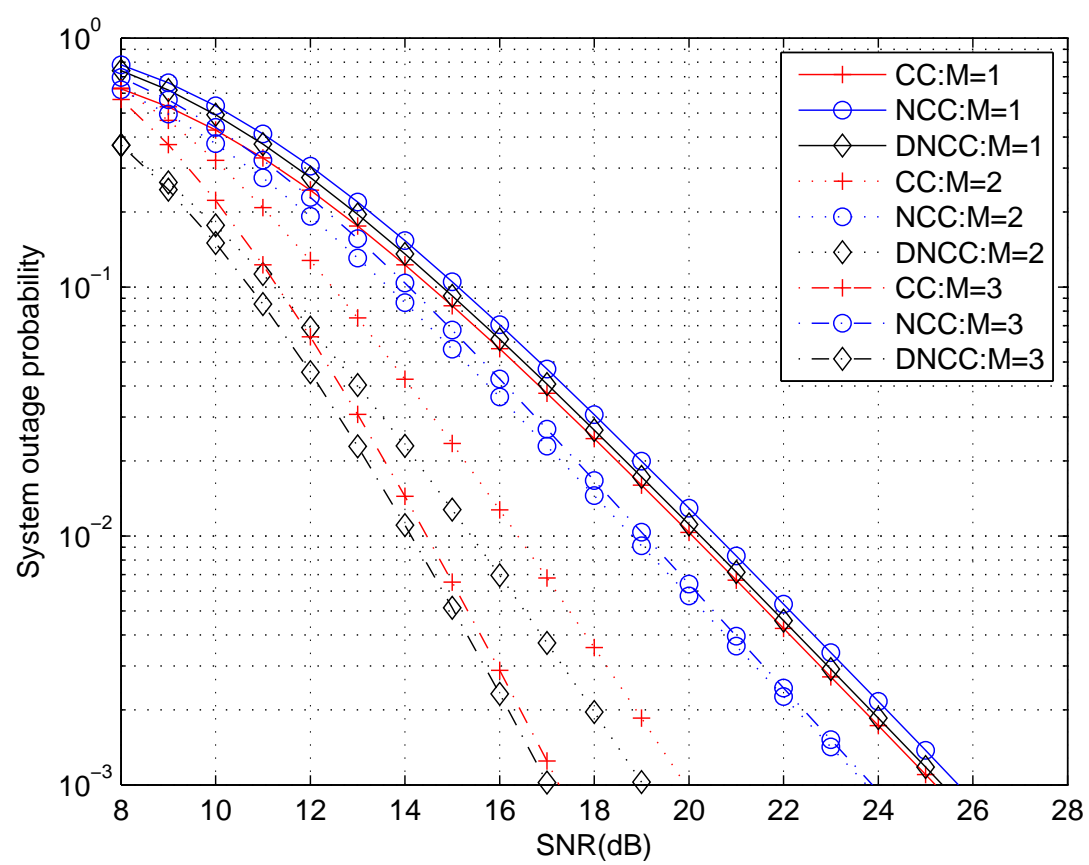

Fig. 5. System outage probability comparison. ( $N=3, M=1, \ldots 3)$

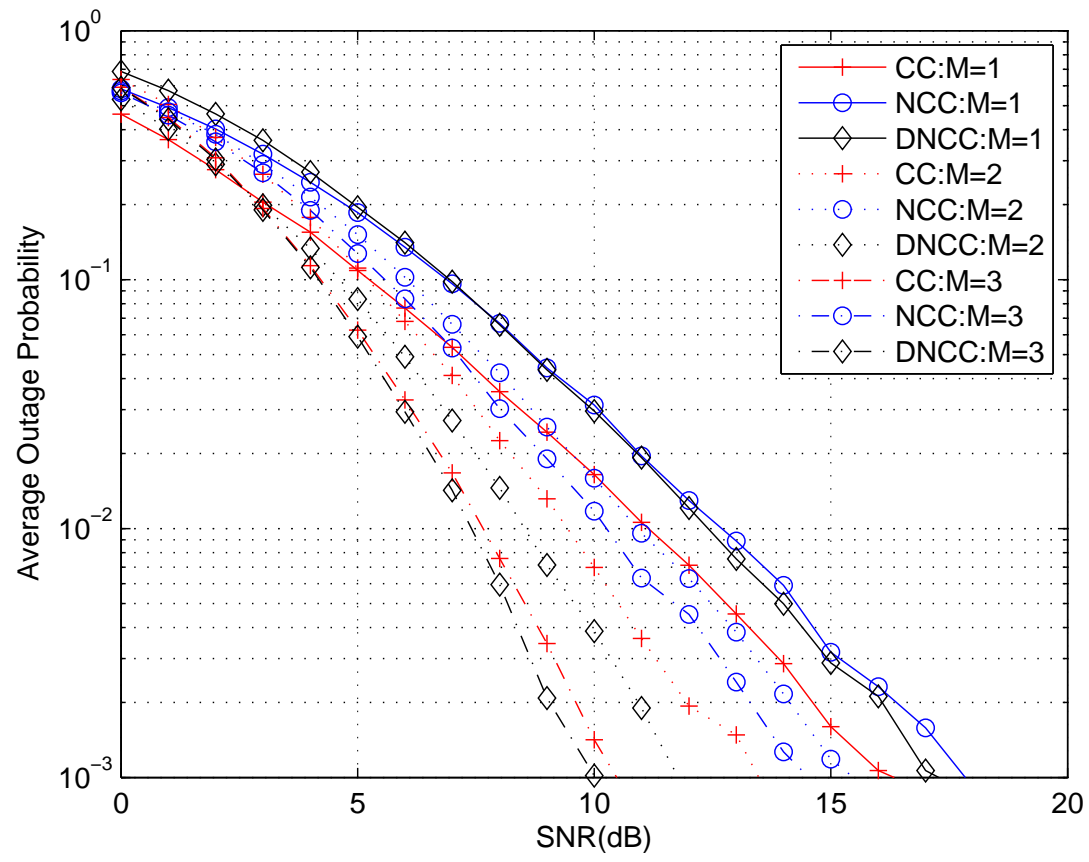

Fig. 6. Average outage probability per destination, $(\mathrm{N}=2, \mathrm{M}=1, \ldots 3)$ 


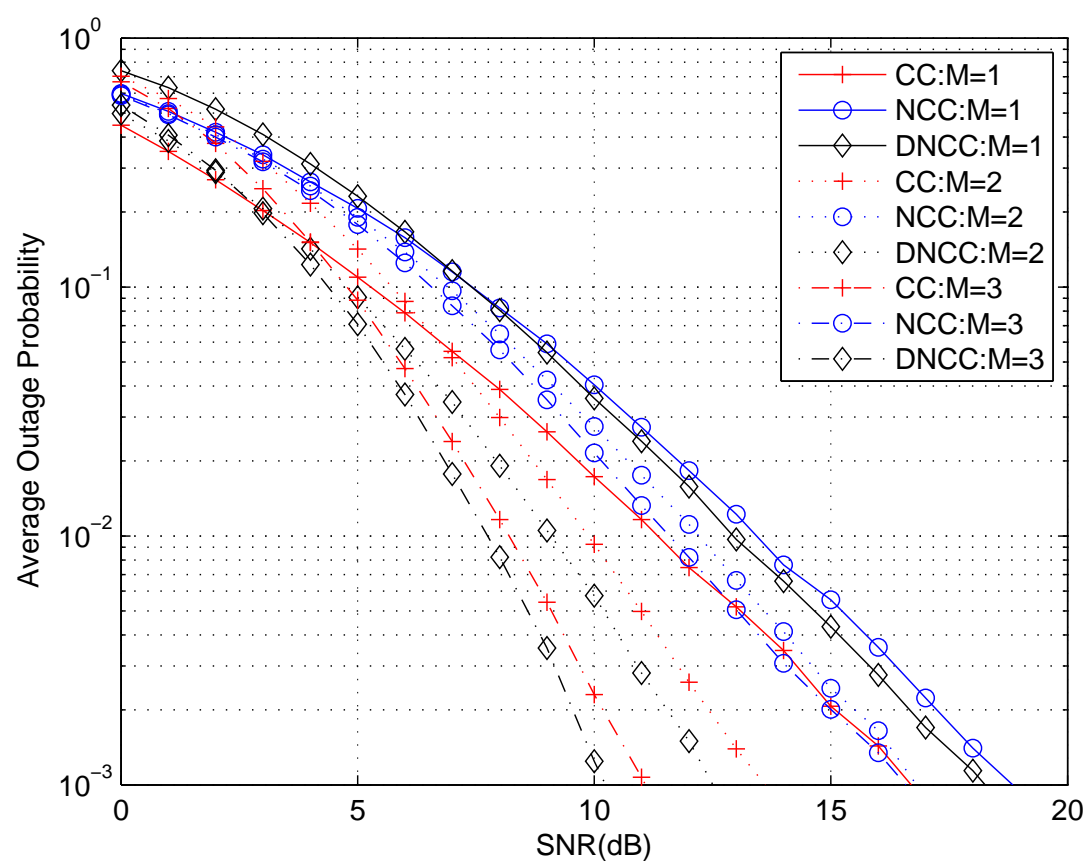

Fig. 7. Average outage probability per destination, $(\mathrm{N}=3, \mathrm{M}=1, \ldots 3)$

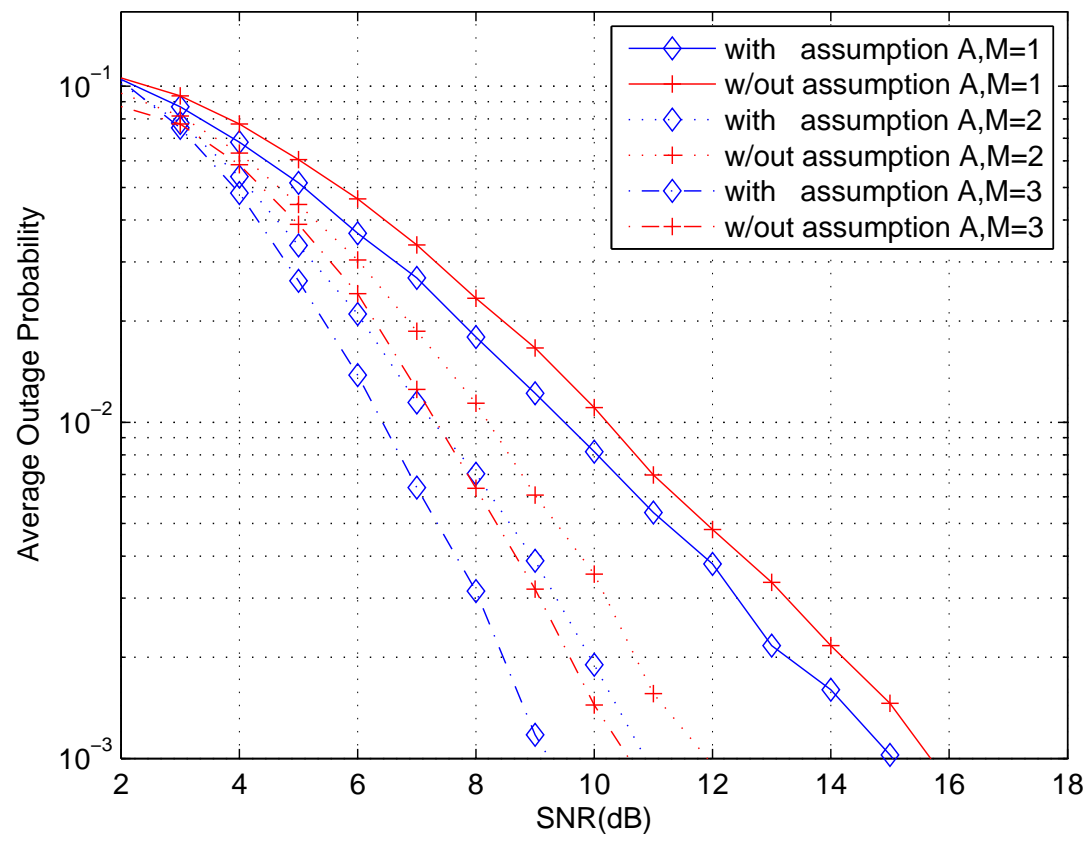

Fig. 8. Average outage probability per destination, $(\mathrm{N}=2 \mathrm{M}=1, \ldots 3)$ 


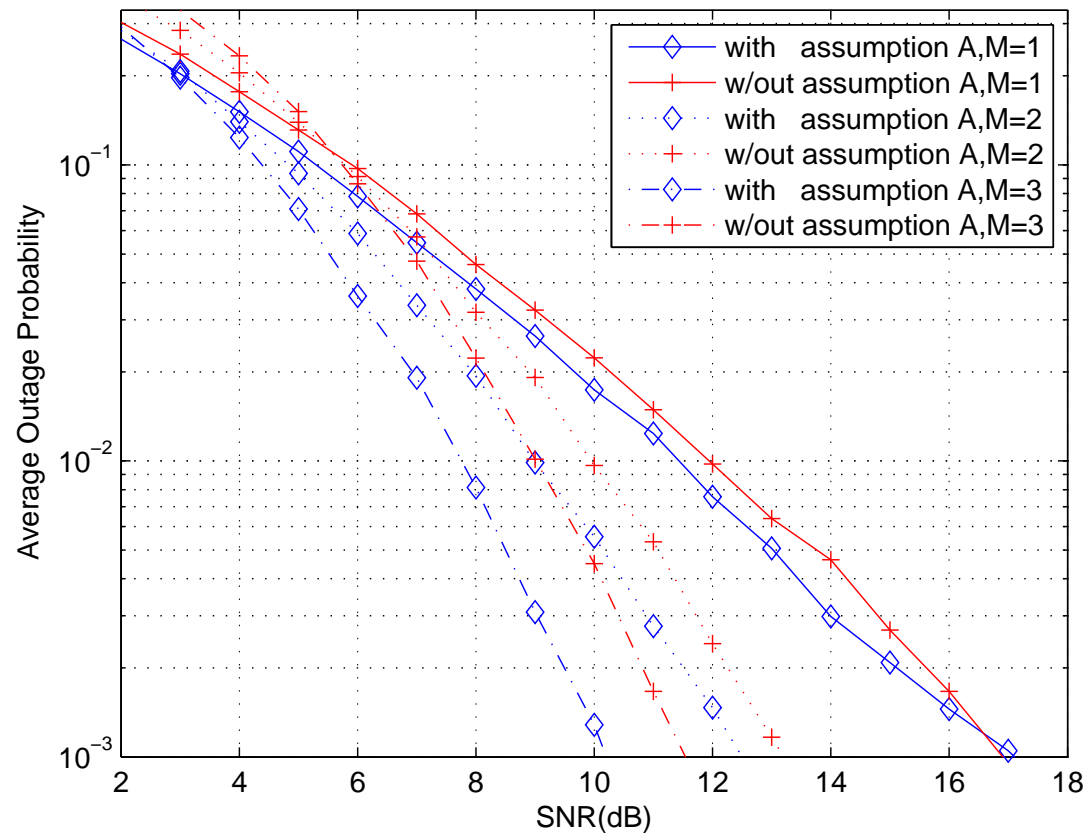

Fig. 9. Average outage probability per destination, $(\mathrm{N}=3 \mathrm{M}=1, \ldots 3)$ 